\title{
Comunicación

\section{Convergencia, emoción y crisis: los periodistas en Puerto Rico evalúan su campo en el siglo XXI ${ }^{1}$}

\author{
Convergence, emotion and crisis: \\ Journalists in Puerto Rico evaluate their field in the XXI Century
}

LOURDES LUGO-ORTIZ2

Este ensayo explora cuáles son los principales retos que enfrenta el periodismo, según los periodistas en Puerto Rico. Mediante el método de la encuesta, identificaron la crisis económica y la entrada de Internet como los retos primarios del campo. Según ellos, éstos no solo inciden en la reestructuración y reducción de las salas de redacción, sino que llevan a una práctica que se distingue por la extrema inmediatez que, en muchas ocasiones, sacrifica la integridad del contenido de los medios.

PALABRAS CLAVE: Convergencia, Internet, periodismo, Puerto Rico, autopercepción.
This article explores the challenges faced by journalists in Puerto Rico. Based on surveys, the work reports that respondents see their trade is being influenced by two main processes: one involves an economic crisis that has had a negative impact on the resources available for newsroom operations. The second involves a drive for immediacy in online news coverage, which often sacrifices the integrity of media content.

KEY WORDS: Convergence, internet, journalism, Puerto Rico, self-perception.

1 Agradezco la lectura cuidadosa y las sugerencias de los profesores Milagros Acevedo Cruz y Mario Roche y de la periodista Laura N. Pérez. Ninguno de ellos, sin embargo, es responsable de las fallas en las que el texto pudiera incurrir.

2 Universidad de Puerto Rico, Puerto Rico.

Correo electrónico: lourdeslugoortiz@gmail.com

P. O. Box 21880, San Juan, Puerto Rico 00931-1880. 
Internet ha revolucionado las formas de acceder, producir y transmitir contenido de corte informativo, lo que ha impactado no solo las estructuras económicas y organizativas de los medios tradicionales, sino también las rutinas profesionales de los periodistas. Este fenómeno de corte global -delimitado principalmente por el grado de conectividad a la red que tiene cada país-, también ha acontecido en Puerto Rico.

La reestructuración de las salas de redacción y la experimentación con diversas estrategias periodísticas para atrapar la atención de la audiencia constituyen algunos de los giros que han moldeado las prácticas de los que hacen la noticia. En tanto sujetos productores de información al interior de las empresas mediáticas en que laboran, el peso de la ejecución de los cambios influidos por la web ha recaído en los hombros de los mismos periodistas. En reconocimiento a que poseen una mirada privilegiada para valorar su campo profesional, este ensayo se dirige a explorar, mediante el método de la encuesta, cuáles, según ellos, son los principales retos que enfrenta el periodismo ya finalizada la primera década del siglo XXI.

\section{MIRADA PANORÁMICA A LOS MEDIOS INFORMATIVOS EN PUERTO RICO}

Las posibilidades del multimedia, de participación y de colaboración ciudadana de Internet han presentado grandes retos a los principales medios en Puerto Rico. Al igual que en muchos otros países, han sumergido a las plataformas informativas en un proceso de convergencia o de integración mediática, en el que la red juega un papel central. Tal y como lo definen Salaverría y Negredo (2008):

Un proceso de convergencia es, por su propia naturaleza, algo siempre inacabado. Cuando esas dos líneas terminan por unirse finalmente en un punto, dejan de ser convergentes. En esa intersección, es donde se sitúa el concepto periodístico de "integración” (p. 51).

Un medio integrado, por lo tanto, es aquel capaz de nutrir de contenidos a dos o más plataformas "mediante una única unidad de producción”. El proceso de convergencia de los medios se da, precisamente, 
al interior de lo que Jenkins (2006, p. 2) ha denominado "la cultura de convergencia" que se caracteriza por el fluir de contenido a través de diferentes plataformas mediáticas, por la cooperación entre múltiples industrias mediáticas y por la conducta migratoria de las audiencias que se mueven hacia el lugar que le brinde el tipo de experiencia de entretenimiento y de información que están buscando, entre ellas la de tipo interactivo. En este sentido, convergencia es una palabra que logra describir los cambios tecnológicos, industriales, culturales y sociales; su uso depende de quién está hablando y sobre lo que está hablando (p. 3).

Insertos en este hacer e influidos por las interacciones que logra con sus públicos, los medios en la isla han ido transformando su cultura periodística, las formas de producir contenido y sus estructuras organizativas. De los cuatro rotativos nacionales, los dos de mayor circulación -El Nuevo Día y Primera Hora, propiedad de la familia Ferré Rangelfueron los primeros en crear sus páginas en Internet, y, desde entonces, se han insertado en el proceso de convergencia como ningún otro medio en la isla. El periódico más importante del país, El Nuevo Día -fundado en 1970 y considerado de récord-, creó su página cibernética en 1998, y su contraparte, Primera Hora -de corte popular y establecido en 1998-, hizo lo propio en 2000. En septiembre de 2011, la familia Ferré Rangel anunció la creación de GFR Media Group, empresa que une ambos periódicos, "así como varios medios digitales y regionales". Como parte de los cambios planificados para 2012, el conglomerado contempla fusionar las salas de redacción de ambos rotativos e incursionar en otras plataformas mediáticas, que incluyen radio y televisión (Custodio Collazo, 2011, p. 54).

Los otros dos periódicos nacionales, El Vocero, fundado en 1970, y Puerto Rico Daily Sun, en 2008, comenzaron a desarrollar sus páginas cibernéticas en los primeros meses de 2010. El primero, a finales de 2010, retomó con fuerza su viejo estilo editorial de enfatizar en historias policíacas y de corte sensacionalista tanto en su plataforma impresa como en la digital. El segundo, Puerto Rico Daily Sun, emana del único periódico en inglés publicado en Puerto Rico desde 1959, The San Juan Star, cerrado en 2008. Meses después, los mismos empleados organizaron una cooperativa, Puerto Rico Daily Sun, y, en noviembre de 2009, crearon su página electrónica. 
Las estaciones televisivas y radiales también han incursionado en el mundo convergente. Por un lado, los tres principales telenoticiarios (Canal 2, 4 y 11) contaban, ya en 2011, con páginas en el ciberespacio en donde se puede acceder a notas informativas y sonidos generados por agencias de noticias, así como a producciones de sus reporteros. Por otro lado, las principales cinco estaciones de radio informativas también han ido adoptando elementos del multimedia en diversos grados. Algunas de ellas, como WKAQ AM y Noti Uno, han diseñado páginas digitales en las que se escucha la programación de las emisoras y se pueden ver vídeos, fotografías y leer notas informativas. Mientras, otras han creado su propia aplicación móvil (Radio Isla 1320) así como cuentan con enlaces a su programación en vivo o en vídeo (WAPA Radio y Radio Isla 1320) y a los archivos de sus trabajos periodísticos (Boricua 740). Mas es de esperarse que, en el futuro cercano, todas las estaciones televisivas y radiales aceleren el proceso convergente.

Internet no solo ha afectado el proceso de producción al interior de las estructuras organizativas, sino la solvencia económica de los medios.

\section{ECONOMÍA Y CAMBIOS}

\section{EN LOS PRINCIPALES MEDIOS}

El golpe que la red ha causado a los medios de comunicación tradicionales alrededor del planeta, particularmente a los impresos, ha sido documentado por diversos académicos (Franco, 2010; Fuller, 2010; Igarza, 2008; Jones, 2009; Meyer, 2004; Salaverría \& Negredo, 2008; Valdettaro, 2009). La pérdida de ingresos publicitarios, la necesidad de crear un modelo económico rentable, así como el progresivo envejecimiento del público representan algunos de los desafíos que enfrentan muchos medios de comunicación tradicionales, al interior de un periodo de crisis económica global. Todo esto se da mientras Internet se va consolidando como un medio de mayor atracción publicitaria (Fuller, 2010; Jones, 2009; Meyer, 2004; Salaverría \& Negredo, 2008; Valdettaro, 2009). Mientras el impacto de la web en Estados Unidos y Europa ha sido considerable, en Latinoamérica ha 
sido menor debido a que la irrupción de Internet en dicha región ha sido más lenta. No obstante, según Franco (2010), será cuestión de tiempo para que la red ejerza la misma presión que ha efectuado en otros lugares.

En Puerto Rico, como en otros países, los medios de comunicación tradicionales, con gran énfasis en el impreso, han recibido una fuerte estocada en sus ganancias. Esto es así pese a que la penetración de Internet en la isla para junio de 2011 alcanzaba 42.6\%, aunque en los últimos años esta cifra ha escalado de forma vertiginosa (Internet World Stats, 2011) y muchos lectores de prensa tradicional han migrado a la red. ${ }^{3}$ Los medios en la isla están enfrentándose a las consecuencias que acarrea que la plataforma cibernética sea gratuita, a la baja rentabilidad de la publicidad en sus páginas $\mathrm{y}$, en el caso del impreso, a la pérdida publicitaria. Esto, a su vez, los ha estimulado a reinventar las formas de allegar mayores públicos, integrando la telefonía móvil, las redes sociales y otros recursos que provee el ciberespacio, como la blogosfera. Sin embargo, en el proceso, han disminuido sus plantillas de empleados -asignando más trabajo a los que permanecen en sus puestos- como estrategia para aminorar los costes de producción. Los principales periódicos en la isla han sufrido grandes pérdidas en su nómina (El Nuevo Día), reducciones salariales y de jornada laboral (El Vocero), incluso hasta cierres (The San Juan Star y la mesa de español de Associated Press). Además, en el caso de la televisión, las constantes reestructuraciones se han manifestado con bastante claridad en los noticiarios, dado que, por los últimos 30 años, es donde se ha centrado la producción nacional. Por ejemplo, en 2009, el noticiario televisivo del Canal 11 expulsó a cerca de 35 empleados y redujo su edición estelar de una a media hora de duración. El noticiario de la estación gubernamental, Canal 6, prácticamente fue eliminado, dejando solo un puñado de su equipo de trabajo, aunque a partir de octubre de 2010 inició la producción de dos telenoticiarios con un reducido personal. A este cuadro, se

3 No obstante, una investigación realizada por Estudios Técnicos con una muestra probabilística de 500 sujetos señala que, para $2010,45 \%$ de la población puertorriqueña estaba conectada a la red. El estudio tiene un margen de error de más menos 4.48 (Custodio Collazo, 2010, p. 35). 
le añade la cantidad de programación local de entretenimiento que se ha suprimido de las estaciones de televisión.

Las formas de generar recursos económicos mediante la publicidad en la plataforma digital de los medios tradicionales continúan siendo un enigma, y no porque Internet no sea rentable. Todo lo contrario, la productividad de la red ha sido más que comprobada, como se ha visto en los casos de Google, Facebook, eBay y Amazon, entre muchos otros. La publicidad cibernética generalmente se pauta en plataformas ajenas a los medios informativos, y la poca que genera no es suficiente para echar hacia adelante económicamente la producción noticiosa o equiparar el nivel de ganancias al que estaban acostumbrados. Tanto es así, que en el caso de Estados Unidos se estima que de cada 10 dólares de ingresos publicitarios, solo uno proviene del ciberespacio (Salaverría $\&$ Negredo, 2008, p. 22). A este cuadro contribuye el problema de que muchos portales se han convertido en free riders informativos, en otras palabras, publican información producida por los medios noticiosos, pero son los portales - como Yahoo y Google, por mencionar dos- los que reciben las ganancias publicitarias que generan la búsqueda, el enlace y la adquisición de la información a través de sus páginas (Fuller, 2010; Jones, 2009).

La pérdida de ingresos ha llevado a que los medios informativos ensayen no solo con los recursos del ciberespacio, sino con la presentación de un contenido melodramático y sensacional, estrategias que datan desde el inicio de la prensa comercial, pero que, ya entrado el siglo XXI, han tomado un nuevo aire.

\section{COMERCIALIZACIÓN Y CONTENIDO EMOTIVO}

El desarrollo de la prensa moderna y comercial en el siglo XX dejó atrás el modelo del periodismo político, y, desde entonces, el mercado se convirtió en el motor que ha impulsado el negocio informativo. Con ello, la publicidad y las ganancias se han conformado en elementos esenciales de la estructura económica de los medios en Estados Unidos, Europa y otros países, incluyendo a Puerto Rico. Este esquema ha impactado tanto las rutinas periodísticas como el producto informativo (Bagdikian, 1983; Hallin, 2000; Herman \& 
Chomsky, 1988; Hoyer \& Lauk 2003; McChesney, 2003; Shoemaker \& Reese, 1996).

$\mathrm{Al}$ interior de esta estructura y como prácticas de extrema comercialización, los medios han adoptado el sensacionalismo y la banalización de los contenidos informativos con el fin de atraer mayores públicos. La adopción de estas técnicas se inició entre finales del siglo XIX y principios del siglo XX, y mostró tal impacto que ya, en 1947, la Comisión Hutchins había identificado el sensacionalismo como una práctica que afectaba la función de la prensa en una sociedad democrática (Commission on the Freedom of the Press, 1947; McChesney, 2003). En la década de los setenta resurgió con la competencia que presentó la televisión a los otros medios, y ha cobrado un tercer aire con la irrupción de Internet. Insertos en ese estilo es que las historias de crímenes, desastres, celebridades y chismes han proliferado en los medios tradicionales y cibernéticos, porque se piensa que atraen a mayores audiencias.

Sin embargo, según Siegel (2008), el sensacionalismo y la banalización del periodismo del siglo XXI ha alcanzado un nuevo nivel, potenciado por los desarrollos tecnológicos. Afirma que el periodismo de Internet toma en cuenta como único criterio de éxito el índice de "popularidad", ya que el énfasis en el despliegue noticioso se determina sobre la base de la cantidad de visitas que tiene la información en determinado tiempo, no por la calidad informativa. Este criterio afecta de igual forma al contenido digital como al impreso. Por un lado, el problema, de acuerdo con Siegel (2008), es que estos actos equiparan "la información con el poder de conocimiento" (p.156). Son rutinas que, al fin y al cabo, llevan a desvalorizar el propio conocimiento y lo convierten en simple información. O, como escribe Bourdieu (1996), las informaciones tendentes a homogeneizar y a banalizar "tienen el efecto de crear un vacío político, de despolitizar o de reducir la vida del mundo a la anécdota y al cotilleo (que puede ser nacional o planetario, con la vida de las estrellas o de las familias reales)" (pp. 65, 74-75). Por otro, gracias a la conectividad con las audiencias, los gestores periodísticos pueden cuantificar las visitas y, con ello, identificar los temas que son de mayor interés para los públicos, ya sea porque son los más visitados o comentados. Esta lógica ha sumado un nuevo criterio de noticiabilidad a la práctica periodística. 
El reapego a las estregias sensacionales y banales, según Fuller (2010), responde a las nuevas formas en que las audiencias consumen la información. El informe anual 2009 del Project for Excellence in Journalism (PEW) describe el perfil de los públicos (prosumidores o prosumistas) del siglo XXI: "They hunt and gather what they want when they want it, use search to comb among destinations and share what they find through a growing network of social media". Lo sensacional y lo emocional se han usado como ganchos para hacer visible cierto contenido ante la cantidad casi ilimitada de alternativas que los potenciales públicos tienen ante sí: "Emotion makes you pay attention ... Emotion makes you remember" (Fuller, 2010, p. 3). El énfasis en lo nuevo y en lo negativo genera mayores emociones que los temas contrarios. Él mismo escribe al respecto: "When a person's information processing capacity is stressed through information overload or multitasking, she is more likely to rely on emotional cues and use social stereotypes in making decisions about another person" (p. 61). Esta es la razón, argumenta el exeditor del Chicago Tribune, por la que las historias de corte sensacionalista, que no son nuevas en el panorama mediático, forman parte, cada vez con mayor frecuencia, de la agenda noticiosa no solo de los tabloides, sino también de muchos medios de récord (Fuller, 2010).

La fluida "tabloidización" de los medios informativos -que se caracteriza por la producción de un contenido sensacionalista orientado hacia el entretenimiento y que asegure altos niveles de gananciasdenota, para algunos, el fracaso de la llamada prensa seria, ya que lo banal tiene la capacidad de darles acceso informativo a ciertos grupos a los que la prensa de récord no llegaba (Hallin, 2000; Jones, 2009; Ornebring \& Jonsson, 2004). No debe sorprender que el periodismo sensacional haya afectado de forma transversal a casi todas las plataformas periodísticas. Para Steimberg (2000), “en las últimas décadas, se ha hecho cada vez más difícil diferenciar los rasgos textuales de los publicaciones tradicionalmente definidas como amarillas o sensacionalistas de aquellas clasificadas como "serias"” (p. 235). Son las historias sensacionales las que llaman la atención, versus el estilo pálido y apagado del periodismo "serio", del cual es fácil ser distraído (Fuller, 2010). Además, en un ambiente de blogueros, noticias de 
cable y de periodismo tabloide, se hace difícil que los medios serios se resistan a las presiones de la competencia y del mismo público de no divulgar cierta información, mucho más cuando el competidor ya lo ha hecho (Jones, 2009).

En este contexto -en el que los medios en Puerto Rico se encuentran sumergidos en un proceso de convergencia que ha afectado la relación con las audiencias, las estructuras económicas y la producción de contenido-, es que este ensayo se dirige a explorar cómo los periodistas en la isla evalúan el estado de su campo profesional.

\section{ACERCAMIENTOS METODOLÓGICOS}

El estudio sobre el rol de los periodistas visto desde los mismos productores de la información cuenta con un caudal investigativo desarrollado principalmente en Estados Unidos y Europa, que se inició de forma consecuente en la década de los setenta, y que, en las décadas subsiguientes, ha sido replicado en otros países alrededor del mundo (Beam, Weaver \& Brownlee, 2009; Canel \& Sánchez Aranda, 1996; Humanes, 1998; Johnstone, Slawski \& Bowman, 1976; Mancini, 1999; Mellado, 2008; PEW,1999; Reese, 2001; Weaver, 1996, 1998, 1999; Weaver, Beam, Brownlee, Voakes \& Wilhoit, 2007). No obstante, en el caso de América Latina, solo $16 \%$ de las investigaciones empíricas producidas de 1960 a 2007 se centran en los "roles profesionales" del periodista, o sea en los estudios que intentan "conocer cuáles son los principios, valores, influencias y actitudes que guían a los periodistas en su comportamiento diario, asociado con la información que generan" (Mellado, 2010, p. 138). En Puerto Rico, la cantidad de estudios empíricos sobre periodistas es escasa. El estudio desarrollado por Mellado indica que solo .4\% de la producción latinoamericana empírica sobre los que hacen la noticia de 1960 a 2007 pertenece a la isla.

Ante ese vacío, este ensayo tiene como objetivo rastrear las percepciones de los periodistas en Puerto Rico sobre su campo en un periodo de transición impulsado por la era digital y la economía. En particular, interesa contestar de acuerdo con los periodistas, ¿cuáles son los principales problemas que enfrenta el campo periodístico?, ¿a qué se debe 
la pérdida de audiencia de los medios informativos?, ¿cómo evalúan el periodismo en la isla?, ¿existen diferencias de opinión cuando se considera el medio en el que laboran (escrito, radial, televisivo o cibernético), el puesto que ocupan y los años que llevan en la profesión? Aunque se reconoce su importancia, está fuera del alcance de este ensayo explorar las diferencias por medios particulares, por estilo (prensa popular o de récord) o por si son propiedades nacionales o extranjeras.

Esta investigación adoptó el diseño de una encuesta producida por The Pew Research Center for the People and the Press titulada: Striking the Balance, Audience Interests, Business Pressures and Journalists (1999), la cual fue adaptada al contexto de la isla. Se encuestó a los periodistas que laboran en los 13 medios noticiosos nacionales más importantes, líderes de opinión en Puerto Rico: los cuatro rotativos con sus respectivas plataformas digitales (El Nuevo Día, Primera Hora, Puerto Rico Daily Sun y El Vocero), las cinco estaciones radiales de noticias (WKAQ-AM, Noti-Uno, Radio Isla, WAPA Radio y Boricua 740) y los cuatro noticiarios televisivos (canales 2, 4, 6 y 11).

A cada medio se le solicitó que sometiera una lista de todos los que producen o colaboran en la creación del contenido periodístico informativo en sus salas de redacción, ya sea a tiempo parcial o a tiempo completo, entiéndase: jefes de sección, editores, reporteros, productores, presentadores y fotoperiodistas. Quienes laboran como colaboradores fueron excluidos del estudio. La población que trabaja en los medios encuestados, según los propios directivos, asciende a 357 periodistas activos.

La encuesta de 43 preguntas -realizada del 1 de marzo al 2 de abril de 2010 - fue enviada por correo electrónico y contestada por 113 periodistas activos. ${ }^{4} \mathrm{El}$ margen de error corregido para una población finita (CFP) de las preguntas oscila entre $+-5.7 \mathrm{y}+-8$ con $95 \%$ de nivel de confianza. ${ }^{5}$

La mayoría de los periodistas que contestaron la encuesta labora para periódicos (77.7\%), para una versión en línea de algún medio (44.7\%), y el resto, para televisión (12.9\%) y radio (4.7\%). ${ }^{6}$ Estos porcentajes

4 Este ensayo se basa solo en algunas de las preguntas del cuestionario.

5 Tamaño de la muestra corregido para una población finita $=1+n /(n-1 /$ pob. $)$.

6 Muchos de los periodistas trabajan en más de un medio informativo; por eso, el porcentaje supera $100 \%$. 
coinciden con el ambiente laboral de la isla, en el que los diarios, con su componente digital, absorben una mayor cantidad de empleados que los otros medios.

Los reporteros son el grupo con mayor representación en el estudio (65\%), a los que les siguen los jefes de sección (22.9\%). Seis de los 13 directores de los medios contestaron la encuesta, lo que equivale a $46 \%$ de ellos. La mayoría de los participantes lleva 13 años o más como periodistas $(46.4 \%)$, siguiéndole en orden descendente los periodistas que tienen experiencia laboral de 8 a 12 años (25\%), de 4 a 7 años $(25 \%)$ y los que llevan tres años o menos en el campo periodístico (3.6\%). Una limitación del estudio gira en torno a la poca representación en la muestra de aquellos con tres o menos años de experiencia o aquellos que fungen como productores, fotoperiodistas y que laboran en la radio, lo que inhibe que se establezca un análisis diferenciado de estas categorías en los resultados aquí presentados.

Se podría trazar el perfil de los encuestados como un reportero o reportera que trabaja en un rotativo o en una versión digital con una experiencia laboral de ocho años o más.

A continuación, se presentan los resultados organizados en cinco secciones, seguidos por las conclusiones.

\section{PROBLEMAS PRINCIPALES DEL PERIODISMO: ECONOMÍA, INTERNET Y CONTENIDO}

Cuando se les preguntó a los periodistas en la isla que expresaran en sus propias palabras cuál es el principal problema que enfrenta el campo periodístico, identificaron, de forma frecuente: la situación económica que atraviesan los medios (44.2\%), el impacto que ha tenido Internet sobre la prensa $(36.5 \%)$ y la pobre calidad del contenido periodístico (27.9\%). ${ }^{7}$ Estos resultados no mostraron diferencias significativas por medio, años de experiencia y puesto que ocupan (véase Tabla 1).

Dentro del problema de la economía, que fue señalado por $44.2 \%$ de los periodistas, los argumentos principales apuntan hacia la crisis

7 Al ser una pregunta abierta, los encuestados identificaron más de una razón; por eso, el porcentaje supera el 100\%. 


\section{TABLA 1}

DESDE SU PERSPECTIVA, ¿CUÁL ES EL PRINCIPAL PROBLEMA QUE ENFRENTA EL CAMPO PERIODÍSTICO?

\begin{tabular}{lc}
\hline & $\%$ \\
\hline $\begin{array}{l}\text { ECONOMÍA: crisis financiera y presiones comerciales, escasez de } \\
\text { puestos periodísticos, eliminación de plazas. }\end{array}$ & 44.2 \\
INTERNET: uso de las nuevas plataformas digitales, convergencia & 36.5 \\
$\begin{array}{l}\text { como responsable de eliminación de plazas, inmediatez. } \\
\text { CONTENIDO: banalización informativa y falta de profundidad, falta }\end{array}$ & 27.9 \\
\begin{tabular}{l} 
de investigación. \\
\hline
\end{tabular}
\end{tabular}
$\mathrm{n}=104$ (+-6.4, 95\% nivel de confianza).

financiera y las presiones comerciales (32.6\%), la escasez de puestos periodísticos (14.4\%) y la eliminación de plazas (11.5\%). ${ }^{8} \mathrm{El}$ aspecto laboral se perfila como uno que preocupa a muchos periodistas.

La crisis económica que viven los medios representa el problema principal para la editora de un rotativo:

La reducción de las salas de redacción, por motivos económicos, baja en pautas publicitarias y aumento en costos de papel. La competencia que los usuarios le suman a la competencia de otros medios.

Mas la crisis económica no solo impacta la supervivencia de los medios -y, como consecuencia, la falta de trabajo y la eliminación de plazas- también afecta la calidad de la información periodística, cuyo énfasis recae en el contenido mercadeable, commodity news. Según una reportera del medio impreso y electrónico, el principal dilema que confrontan es:

La crisis económica. Aunque la mayor parte del tiempo intentamos ignorar este hecho, los medios de comunicación son negocios y las presiones sobre el periodismo se empiezan a sentir más a medida que el impacto económico es

8 Algunos periodistas mencionaron más de un problema económico; por eso, la suma de los tres argumentos principales supera $44.2 \%$. 
mayor. La reducción de espacio y tiempo, la eliminación de plazas, la competencia por tener "la noticia que vende" relegando la calidad periodística son todos efectos negativos de la tan sonada recesión.

O, como dice un editor y jefe de sección de un rotativo, la crisis del modelo económico prevaleciente constituye el principal problema, ya que ha puesto en riesgo la supervivencia del periodismo serio, o apegado a los valores de la responsabilidad social:

La crisis que enfrenta el modelo económico de la industria periodística ha causado el cierre de múltiples talleres, el virtual desmantelamiento de otros $\mathrm{y}$, en términos generales, la reducción dramática de opciones informativas y en la calidad de los que quedan en pie, los cuales, a menudo, banalizan sus contenidos para intentar sobrevivir en un clima económico poco menos que catastrófico.

Los problemas con el contenido periodístico surgen como consecuencia del intento de sobrevivir económicamente y de la extrema comercialización de los medios. Para los encuestados, las deficiencias que presenta el contenido (27.9\%) se identifican en la banalización informativa $(9.6 \%)$, como el editor antes mencionado, y en la falta de profundidad e investigación (23.1\%). 9

La reducción de plazas, la competencia con otras plataformas, entre otros, crean superficialidad en el contenido. Apunta una presentadora reportera de televisión:

[L]a crisis económica ... menos empleados, periodistas redactores ... para cubrir igual cantidad de historias incluso para distintas versiones del mismo medio ... el resultado es superficialidad. Por otro lado, la competencia que precisamente provocan la entrada de nuevas opciones de información para los ciudadanos, a veces provoca que se sacrifique la calidad periodística.

9 Algunos periodistas mencionaron más de un problema de contenido; por eso la suma de los dos argumentos principales supera $27.9 \%$. 
La baja calidad periodística -consecuencia de las presiones económicas y de nuevas opciones informativas, como las que presenta Internet- también es resultado de las presiones de los anunciantes, lo que, para un reportero de prensa escrita, impacta la libertad de expresión - ¿o la libertad de prensa?- y se convierte en el problema principal que enfrenta el campo:

La libertad de expresión. Al presente, la presión de parte de los dueños de los medios por no molestar a sus anunciantes ha hecho que los periodistas no puedan hacer investigaciones profundas. Toda noticia que pueda afectar a sus grandes anunciantes es, en ocasiones, no publicada.

Para este periodista, el problema principal reside en que las presiones comerciales, en un periodo de crisis económica, afecta el papel que tiene la prensa en una sociedad democrática: proveer información para que la gente tome decisiones acertadas en sus vidas (Commission on the Freedom of the Press, 1947; Kovach \& Rosenstiel, 2003).

La postura de los dos reporteros mencionados anteriormente no es aislada. Cabe destacar que, cuando se les aborda directamente con una pregunta cerrada, los periodistas en la isla perciben las influencias de los anunciantes entre "mucha" y "bastante" en $46.2 \%$ ("mucho", 16.1\% y "bastante", 30.1\%) (Lugo-Ortiz, en prensa). Esto coincide con los hallazgos en otros países. Por ejemplo, en un estudio realizado en Argentina, los periodistas reportan, de forma mayoritaria $(69.5 \%)$, la presión del departamento comercial en el contenido que producen (Giacobbe, 2005). También, en investigaciones más recientes, $30 \%$ de los periodistas en Estados Unidos identifican algún tipo de presión comercial (Beam, Weaver \& Brownlee, 2009; Weaver et al., 2007).

A la economía de los medios y el impacto que tiene sobre el ámbito laboral y los contenidos, se le suma Internet como responsable de una de las principales dificultades que enfrenta el campo (36.5\%), según las propias palabras de los periodistas. Al interior de este argumento, mencionan: el uso que los medios informativos le están dando a esta plataforma (23.1\%), la convergencia como responsable de la eliminación de plazas $(11.5 \%)$, que también constituye un señalamiento al 
interior de los problemas económicos, y la inmediatez que exige esta plataforma (8.7\%). ${ }^{10}$

Para una reportera de negocios, el uso que se le da a las nuevas tecnologías representa el principal problema que enfrenta el campo:

Pienso que hay desconocimiento sobre el uso de las nuevas plataformas de distribución de la información, lo cual causa un conflicto entre la inmediatez y la calidad periodística, en particular en la prensa escrita.

El uso irresponsable de las nuevas tecnologías no solo hiere la excelencia periodística, sino que lesiona los valores de la profesión, lo que constituye el principal problema para una supervisora de la plataforma cibernética:

Sacrificar la veracidad de la información y de las fuentes a cambio de la entrega inmediata y querer convertirse en el primer medio en publicar la noticia.

$\mathrm{O}$, como escribe una reportera de prensa escrita e Internet:

La inmediatez y la transición de convertirse en multimedios, en ocasiones, compromete la calidad del trabajo.

El apego a la verdad, según las periodistas antes mencionadas, queda en un segundo plano por la misma inmediatez: hay que publicar la historia lo antes posible. La competencia en ser los primeros trae como baja la verdad en la producción del contenido multimedia, y esto se da particularmente para los que laboran en prensa escrita. Escribe un reportero:

La falta de profundidad por la presión de tiempo. Hay temas que los compañeros de radio y TV dicen son difíciles para tocar en sus medios, por lo que no los tocan, y en prensa escrita ahora estamos tratando de redactar para dos plataformas distintas, entiéndase el periódico e Internet.

10 Algunos periodistas mencionaron más de un problema relacionado con Internet; por eso, la suma de los tres argumentos principales supera $36.5 \%$. 
La prisa por publicar la información, además, viene a consecuencia de la competencia que generan las plataformas ajenas al periodismo en la red. Según una editora de contenidos de la plataforma digital:

El principal reto del periodismo es la manera adecuada de cómo llevar la información de manera rápida e íntegra. Hoy día, nuestra principal competencia son las redes sociales, los blogs y el periodismo ciudadano. Ahora, cualquiera puede ser periodista o, mejor dicho, puede publicar la noticia con menos procedimientos y menos burocracia que un medio noticioso.

La inmediatez, la competencia de las redes sociales y el contenido generado por los usuarios representan un gran reto para los medios informativos. La participación de la ciudadanía o la proliferación del "neoperiodismo", como escribe Igarza (2008), "se sitúa en las fronteras de los medios y actúa diversificando las fuentes de información y, a la vez, completando los medios de comunicación institucionalizados" (p. 27). Ya el ciudadano no depende únicamente de los medios periodísticos para adquirir información, usa otras plataformas, lo que para muchos periodistas en la isla se articula como uno de los principales desafíos del campo periodístico.

\section{CRÍTICAS VÁLIDAS: SUPERFICIALIDAD Y ERRORES EN EL CONTENIDO}

Los periodistas encuestados no tienen problema alguno en articular en sus propias palabras que la economía e Internet son los dos principales problemas que enfrenta el campo periodístico, los que, a su vez, hieren la producción del contenido. Entonces, cuando se les presentó una lista de posibles críticas que se le dirigen a la prensa, tampoco vacilaron en catalogar como "válidos" muchos señalamientos relacionados con la pobre calidad de la información periodística.

La superficialidad y los errores en el contenido son las dos principales críticas válidas que los encuestados seleccionaron, a las que se les suma la competencia interna y externa del quehacer de los mismos medios. Particularmente, priman como críticas válidas que el quehacer periodístico le presta muy poca atención a los asuntos complejos (74.3\%), los informes 
periodísticos están plagados de errores $(72 \%)$, los jefes no dedican suficiente tiempo en corregir los errores $(71.3 \%)$, la distinción entre noticia y el comentario se ha desvanecido $(70.3 \%)$ y el contenido periodístico es superficial (68.3\%). Señalaron, así mismo, la competencia entre ellos mismos como una crítica válida, o sea que los periodistas están obsesionados con las dinámicas internas, como en ser los primeros en cubrir una historia e impresionar a sus colegas (56.4\%) (véase Tabla 2). Estos resultados fueron similares, independientemente de los años de experiencia, no así por el puesto que ocupan o el medio en el que trabajan. Cuatro de seis directores perciben como una crítica inválida que los jefes no le dedican tiempo ni espacio a corregir errores; mientras que, para $100 \%$ de los encuestados que laboran en televisión, figura como una crítica válida. Los directores se exhiben como defensores del funcionamiento de las estructuras organizativas, mientras los que laboran en televisión perciben poca supervisión directa en sus rutinas de producción.

\begin{tabular}{|c|c|}
\hline $\begin{array}{c}\text { TABLA } 2 \\
\text { ALGUNAS CRÍTICAS QUE SE LE HACEN } \\
\text { AL PERIODISMO, ¿CUÁN VÁLIDAS O NO SON? }\end{array}$ & \\
\hline & $\begin{array}{l}\text { Crítica } \\
\text { válida \% }\end{array}$ \\
\hline La prensa les presta muy poca atención a los asuntos complejos. & 74.3 \\
\hline $\begin{array}{l}\text { Los informes de la prensa están cada vez más plagados } \\
\text { de errores. }\end{array}$ & 72.0 \\
\hline $\begin{array}{l}\text { Los(as) jefes(as) no dedican suficiente tiempo ni espacio a } \\
\text { corregir los errores. }\end{array}$ & 71.3 \\
\hline La distinción entre noticia y comentario se ha desvanecido. & 70.3 \\
\hline El contenido periodístico es superficial. & 68.3 \\
\hline $\begin{array}{l}\text { Los(as) periodistas están cada vez más obsesionados(as) con } \\
\text { las dinámicas internas, como en ser los(as) primeros(as) en } \\
\text { cubrir una historia e impresionar a sus colegas. }\end{array}$ & 56.4 \\
\hline
\end{tabular}

Las fallas en el contenido periodístico así como la extrema competencia son críticas válidas que describen el periodismo nacional. Am- 
bas, a todas luces, inciden en el papel del periodismo en la sociedad, ya que violentan el apego a la verdad y, con ello, la disciplina de la verificación y el compromiso con la ciudadanía.

Incluso, la autocrítica es tan fuerte que los periodistas en la isla entienden que los medios están yendo demasiado lejos en la dirección del entretenimiento (76.9\%), independiente de la posición que ocupan, años de experiencia y medio en el que trabajan. La excesiva espectacularización informativa se propone como un problema del contenido periodístico nacional que choca con el llamado a la responsabilidad social que debe cumplir la prensa, según ha sido esbozado por la Comisión Hutchins (Commission on the Freedom of the Press,1947) y Kovach y Rosenstiel (2003). Lo interesante es que, cuando se miran los hallazgos del primer estudio realizado en la década de los setenta sobre los roles de los periodistas estadounidenses, ya éstos mostraban preocupación no solo por lo que cubrían los medios sino por cómo lo cubrían y, entre ello, resaltaron el énfasis en lo sensacional (Johnstone et al., 1976).

Pese a que los encuestados en la isla reconocen que los medios están yendo demasiado lejos en la dirección del entretenimiento, cuando se les pregunta cuán bien el ejercicio periodístico otorga un justo balance entre lo que la gente necesita saber y lo que quiere saber, las contestaciones están divididas: 53\% lo evalúa de "bien" a "excelente", y el restante de "regular" a "mal". No hay mayoría que coincida en que el periodismo en Puerto Rico cumple con proveer la información que la ciudadanía necesita para tomar decisiones adecuadas en un sistema democrático. Como establece Fuller (2010), para cumplir con la misión social del periodismo se requiere que los medios le den a la gente lo que necesita saber pero, a la misma vez, no darle lo que quiere es una receta para el fracaso periodístico. En otras palabras, el reto que enfrentan los medios es cómo concilian las exigencias del público y las del mercado sin renunciar a unos valores profesionales.

El sensacionalismo y la baja calidad del contenido periodístico no son críticas nuevas. Incluso, Henry Luce, cofundador y editor de la revista Time, denunció, ya en la década de los treinta, el peligro que constituye darle a la gente solo lo que quiere. Según el periodista, el resultado de estas acciones es un sensacionalismo craso y una vulgaridad rampante que crea "un incentivo financiero enorme para publi- 
car tonterías ... millas cuadradas de mondongo periodístico" (citado en Jones, 2009, p. 42). Es este el mismo problema de la superficialidad informativa que continúan enfrentando los medios 80 años después y que los periodistas de la isla señalan como un serio obstáculo para que el periodismo logre su misión.

Precisamente, de acuerdo con Entman (2005), lo que distingue el periodismo tradicional del tabloide, del político o del de entretenimiento, es cuán atado cada uno está a las normas periodísticas: precisión, balance, vigilancia del poder y separación entre contenido y publicidad. A esta lista, se le añade la determinación que tienen como empresas en generar ganancias. Todos los medios tienen como objetivo esta última, pero lo que los diferencia entre sí es el grado con el que se apegan a cada uno de los estándares antes mencionados. En el caso de los tabloides, su prioridad principal es maximizar las ganancias, aunque tengan que sacrificar los otros valores (Entman, 2005). Y esa es la ruta que muchos medios están tomando, según los productores de la información encuestados. Sin embargo, al hablar sobre la importancia social de su trabajo, su mirada se suaviza.

\section{TRABAJO QUE HACEN:}

COMPROMISO CON LA PROFESIÓN

Pese a que los periodistas son bastante críticos del contenido que produce la prensa, cuando se les pregunta sobre el resultado neto de su trabajo y sobre si la prensa cumple con ciertas funciones sociales, se sienten más interpelados a evaluarla de forma positiva.

Los encuestados tienden a afirmar, de forma mayoritaria, que el trabajo que la prensa hace es de "bueno" a "excelente" en cuanto a evaluar asuntos importantes de forma interesante (61\%). Esto es así independientemente del lugar en que trabajan, los años de experiencia y el puesto que ocupan. Así mismo, cuando se les presentan dos afirmaciones contrarias para escoger, seleccionan aquellas que evalúan de forma positiva a la prensa: piensan que, generalmente, la prensa publica información correcta (99\%) y está dispuesta a admitir errores (50.5\%) (véase Figura 1). Pese a las faltas en el contenido y la banalización informativa, creen que la prensa hace un trabajo que apela a la audiencia, generalmente se apega 
a la verdad y es confiable. A esto se le añade que los periodistas identifican como una fortaleza el compromiso que tienen con su público y con su profesión. En ese sentido, dicen que le importa la gente sobre quien reporta (64.9\%) y le importa el trabajo que hacen $(87.1 \%)$.

\section{FIGURA 1}

¿CREE QUE SU MEDIO GENERALMENTE TIENDE A PRESENTAR

INFORMACIÓN CORRECTA O CREE QUE LAS HISTORIAS USUALMENTE SON POCO PRECISAS E INCORRECTAS?

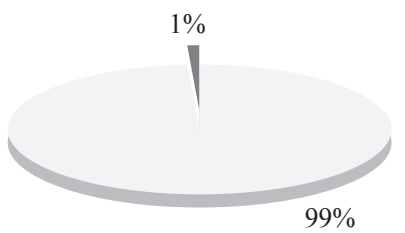

Generalmente publica historias incorrectas Generalmente publica información correcta

+-6.9, 95\% nivel de confianza.

Tanto es así que, además, creen que la prensa cumple con uno de sus objetivos vitales: vigilar al poder. La mayoría de los encuestados piensa que la prensa ejerce presión para que los políticos hagan el trabajo que tienen que hacer $(85.6 \%)$. Internet afecta el contenido periodístico, como ellos mismos afirmaron, pero el rol de servir como vigilantes del poder sigue intacto.

Aunque identifican el esfuerzo de llevar a la audiencia un contenido confiable, que les importa el trabajo que hacen y que afirman su rol como vigilantes del poder estatal y comercial, las opiniones de los encuestados están divididas en cuanto a si la prensa se encuentra o no en declive ( $47.8 \%$ en declive, $36.9 \%$ en ascenso y $15.2 \%$ ninguna aplica). Mas, al analizar por años de experiencia, medio y puesto que ocupan, se presentan unas marcadas diferencias de opinión. Para $70.3 \%$ de los periodistas con 13 años o más de experiencia, la prensa se encuentra en declive. Aquellos que han vivido otros periodos tienden a ver el estado actual de forma pesimista. No es así para aquellos con experiencia entre 8 y 12 años $(52.4 \%$ en ascenso y $28.6 \%$ en declive), para los editores (56.5\% en ascenso y $39.1 \%$ en declive) y para aquellos que trabajan en Internet (50\% en ascenso y $30.6 \%$ 
en declive), quienes en su mayoría perciben que está en ascenso. En otras palabras, los que trabajan en la plataforma digital, que supervisan a otros periodistas y que están en un término medio de su carrera ven el panorama más brillante.

Pese a las críticas dirigidas al contenido periodístico, los periodistas encuestados afirman el lugar de la prensa: tiende a llevar un contenido confiable a la audiencia, le importa el trabajo que hace y cumple su rol de vigilantes del poder. Defienden la labor del periodismo, aunque señalen serias deficiencias en su contenido. Existe un desfase entre la percepción teórica de lo que es su profesión y la apreciación de su práctica profesional. No obstante, las opiniones de los periodistas están divididas en cuanto a si la prensa se encuentra o no en declive.

\section{PÉRDIDA DE AUDIENCIA: INTERNET}

La pérdida de audiencia es uno de los grandes problemas que enfrentan muchos medios de comunicación. Entonces, se les preguntó a los encuestados a qué atribuyen dicha pérdida. Éstos no vacilaron en identificar, de forma mayoritaria, a Internet como responsable de este fenómeno.

Se les pidió a los periodistas que, de una lista, identificaran cuáles de las afirmaciones presentadas constituyen "la razón principal", "razón de mucho peso", "razón de poco peso" o "no es una razón" para la pérdida de audiencia de los medios. Las razones principales o de mucho peso que eligieron -sin que se mostraran diferencias significativas por medio, puesto que ocupan y años de experienciafueron las siguientes: $90.6 \%$ piensa que las plataformas informativas especializadas permiten que la gente obtenga solo la información que le importa (41.2\% "razón principal” y 49.4\% "razón de mucho peso") y $88.3 \%$, que el público tiene cada vez más acceso directo a diversas fuentes de información (37.7\% "razón principal” y 50.6\% "razón de mucho peso"). Ambas afirmaciones están directamente relacionadas con el acceso informativo que provee la red, aunque se debe aclarar sobre el primer punto que el uso selectivo de los medios es una práctica que antecede a Internet y que ha estado presente desde el inicio de la prensa. Además de que los públicos pueden acceder a fuentes directas, 67\% (16.9\% "razón principal” y 46.9\% "razón de mucho 
peso") atribuye el declive a que la prensa no les da suficiente atención a las historias que son significativas para el ciudadano promedio.

Para los periodistas encuestados, la pérdida de audiencia se debe a dos razones principales que interactúan entre sí: el público puede acceder a información de su interés sin requerir la mediación de la prensa, y el periodismo no ajusta su contenido para que sea de provecho a la ciudadanía.

Estos resultados, a todas luces, llevan a pensar sobre el progresivo envejecimiento de los medios tradicionales, particularmente del medio impreso (Jones, 2009; Meyer, 2004; Salaverría \& Negredo, 2008). Un estudio realizado en Estados Unidos revela que, ya en 2008, solo 19\% de las personas entre 18 y 34 años leían el periódico en su versión impresa, y otras tantas investigaciones demuestran la dependencia a la red entre los usuarios jóvenes como fuente principal informativa (Alterman, 2008). En España, el lector promedio de la prensa comercial supera la media de los 43 años y en Inglaterra, los ingleses entre 15 y 24 años aseguran que, luego de que comienzan a usar Internet, dedican $30 \%$ menos de su tiempo a leer los periódicos (Salaverría \& Negredo, 2008). Así mismo, un estudio global conducido en 2005, evidencia que $44 \%$ de los jóvenes prefiere la red antes que ver televisión (Igarza, 2008).

En este contexto, el envejecimiento de la audiencia combinado con que la prensa no ajusta su contenido para que sea de interés a la ciudadanía presenta un reto para la atracción de públicos jóvenes, quienes generalmente son nativos digitales y están acostumbrados a buscar información en las redes sociales o en otras páginas sin tener que depender del periodismo. Sobre esto, Jenkins (2006) sugiere que el contenido periodístico debe adoptar "tácticas juguetonas" que pueda atraer a esa generación joven que se ha criado en un mundo en el que las técnicas participativas de los medios han sido un lugar común para ellos.

NOTA A LA PRENSA: ENTRE "B" Y “C”

En este contexto en el que los periodistas en la isla no vacilan en identificar problemas de contenido y el reto que presenta la plataforma cibernética al quehacer periodístico, se les pidió que calificaran la labor de 
la prensa de Puerto Rico en el último año. La mayoría la calificó entre "bueno" ("B", 41.7\%) y "regular" ("C"C, 45.2\%).11

No obstante, se presentaron diferencias de criterio a la luz del puesto que ocupan los encuestados y los años de experiencia. Cuatro grupos tienden a evaluar a la prensa de la isla mayormente con una " $\mathrm{C}$ ": aquellos que tienen experiencia laboral de 8 a 12 años $(52.4 \%$ " $\mathrm{C}$ " y $38.1 \%$ "B"), los directores (66.7\% o 4 directores la calificaron con " $\mathrm{C}$ " y $33.3 \%$ o 2 directores, con “B”), jefes de sección (52.6\% “C” y 36.8\% “B”) y los supervisores (51.7\% “C” y 37.9\% “'B”). A todas luces, las personas que están en los puestos directivos y de supervisión ven un espacio para alcanzar mayor calidad. Mas en términos generales, los periodistas son cautos en celebrar la excelencia periodística en la isla.

\section{CONCLUSIONES}

Finalizada la primera década del siglo XXI y en un contexto de transformaciones y cambios que inciden en la producción y en el consumo noticioso, así como en el negocio mediático; este estudio evidenció que muchos periodistas en Puerto Rico perciben Internet y la economía como los principales retos que enfrenta el campo periodístico, los cuales afectan de forma evidente la calidad del contenido informativo. La oferta amplia y gratuita de la red se postula como responsable por la reducción de la audiencia y la inestabilidad financiera de los medios. Pese a este cuadro de fuerte autocrítica, los encuestados afirman la importancia de la prensa: evalúa asuntos importantes de forma interesante, le importa el trabajo que hace y la gente sobre quienes reporta. Piensan, además, que cumplen su rol como vigilantes del poder.

Aunque son críticos de las rutinas profesionales que el negocio periodístico impulsa -la extrema comercialización que puede llevar a la banalización informativa y al desapego de la verdad-, los periodistas en la isla siguen percibiéndose como profesionales comprometidos con su trabajo. En otras palabras, apuestan a la importancia y al impacto social

11 El sistema de calificación que se utiliza en Puerto Rico es el siguiente: "A" (excelente o muy bueno"), “B” (bueno), “C” (regular), "D” (deficiente) y "F" (fracasado). 
de su profesión como uno de los valores fundamentales de la práctica periodística moderna, pero señalan unas influencias comerciales indebidas que tienen serias consecuencias en el funcionamiento de la prensa en un sistema democrático.

El reto que tienen los periodistas en la isla ante sí es traducir el apego que dicen tener a su responsabilidad ciudadana en acciones concretas dentro de las estructuras de producción de los medios y, de esta forma, incidir en un contenido más responsable.

\section{Bibliografía}

Alterman, E. (2008, 31 de marzo). Out of print. The New Yorker, p. 49. Bagdikian, B. (1983). The media monopoly. Boston, MASS, EE.UU.: Beacon Press.

Beam, R. A., Weaver, D. H. \& Brownlee, B.J. (2009). Changes in professionalism of U.S. journalists in the turbulent Twenty-First Century. Journalism and Mass Communication Quarterly, 86 (2), 277-298.

Bourdieu, P. (1996). Sobre la televisión. Barcelona, España: Anagrama. Canel, M. J. \& Sánchez-Aranda, J. J. (1999). La influencia de las actitudes profesionales del periodista español en las noticias. Análisis, $23,151-170$.

Commission on the Freedom of the Press (1947). A free and responsible press. A general report on mass communication: Newspapers, radio, motion pictures, magazines, and books. Illinois, EE.UU.: The University of Chicago Press.

Custodio Collazo, M. (2010, 14 de mayo). Sorprende la conexión. El Nuevo Día, pp. 34-35.

Custodio Collazo, M. (2011, 15 de septiembre). Surge potente empresa periodística. El Nuevo Día, p. 54.

Entman, R. M. (2005). The nature and sources of news. En G. Overholser \& K. Hall Jamieson (Eds.), The Press (pp. 48-65). Londres, Inglaterra: Oxford University Press.

Franco, G. (2010). Efectos de las tecnologías digitales en el periodismo de América Latina y el Caribe: ¿qué ha cambiado hasta ahora y qué transformaciones vienen? Sala de Prensa, 129, agosto, XI (6). Recuperado el 2 de febrero de 2011 de http://www.saladeprensa.org Fuller, J. (2010). What is happening to news? The information explo- 
sion and the crisis in journalism. Chicago, IL, EE.UU.: The University of Chicago Press.

Giacobbe, J. y Asociados (2005). Encuesta FOPEA (Foro de periodismo argentino). Recuperado el 13 de noviembre de 2010 de http://www. fopea.org/Etica/Encuesta_sobre_Periodismo

Hallin, D. C. (2000). Commercialism and professionalism in the American news media. En J. Curran \& M. Gurevitch (Eds.), Mass Media and Society (pp. 218-237). Londres, Inglaterra: Arnold.

Herman, E. S. \& Chomsky, N. (1988). Manufacturing consent: The political economy of the mass media. Nueva York, EE.UU.: Pantheon Books. Hoyer, S. \& Lauk, E. (2003). The paradoxes of the journalistic profession: An historical perspective. Nordicom Review, 24 (2), 3-18.

Humanes, M. L. (1998). La profesión periodística en España. Revista Zer, Revista de Estudios de Comunicación, 4 Mayo. Recuperado el 12 de enero de 2011 de http://www.ehu.es/zer/zer4/humanes12.html Igarza, R. (2008). Nuevos medios: estrategias de convergencia. Buenos Aires, Aregentina: La Crujía.

Internet World Stats: Usage and Population Statistics (2011). Internet Usage Statistics: The Internet Big Picture World Internet Users and Population Stats. Recuperado el 15 de mayo de 2012 de http://www. internetworldstats.com/carib.htm

Jenkins, H. (2006). Convergence culture: Where old and new media collide. Nueva York, EE.UU.: New York University Press.

Jones, A. S. (2009). Losing the news: The future of the news that feeds democracy. Nueva York, EE.UU.: Oxford University Press.

Johnstone, J. W., Slawski, E. J. \& Bowman, W. W. (1976). The news people: A sociological portrait of American journalists and their work. Urbana, IL, EE.UU.: University of Illinois Press.

Kovach, B. \& Rosenstiel, T. (2003). Los elementos del periodismo. Madrid, España: Ediciones El País.

Lugo-Ortiz, L. (En prensa). Entre las presiones del público, los dueños y los anunciantes: los periodistas en Puerto Rico miran su campo en el siglo XXI. Revista del Instituto de Cultura Puertorriqueña.

Mancini, P. (1999). La paradoja de los periodistas italianos: difusores teóricos, pero defensores prácticos. Problemas metodológicos e interpretativos. Comunicación y Sociedad, XII (2),115-134. 
McChesney, R. W. (2003). The problem of journalism: A political economic contribution to an explanation of the crisis in contemporary US journalism. Journalism Studies, 4 (3), 299-329.

Mellado Ruiz, C. (2008). Indicadores de identidad y perfil del periodista regional en Chile. Opción, 24 (55), 145-167.

Mellado Ruiz, C. (2010). Análisis estructural de la investigación empírica sobre el periodista latinoamericano. Comunicación y Sociedad, 13. Nueva época, enero-junio, 125-147.

Meyer, P. (2004). The vanishing newspaper: Saving journalism in the information age. Columbia, SC, EE.UU.: University of Missouri Press.

Ornebring, H. \& Jonsson, A. M. (2004). Tabloid journalism and the public sphere: A historical perspective on tabloid journalism. Journalism Studies, 5 (3), 283-295.

PEW-Research Center for the People \& the Press (1999, 30 de marzo). Striking the balance, audience interest, business pressures and journalists' values. Recuperado el 20 de febrero de 2012 de http:// people-press.org/report/67/striking-the-balance-audience-interestsbusiness-pressures-and-journalists-values.

PEW-Project for Excellence in Journalism (2009). The state of the news media: An annual report on American journalism. Recuperado el 24 de febrero de 2010 de http://stateofthemedia.org/2009/

Reese, S. (2001). Understanding the global journalist: A hierarchy-ofinfluences approach. Journalism Studies 2 (2),173-187.

Salaverría, R. \& Negredo, S. (2008). Periodismo integrado: convergencia de medios y reorganización de redacciones. Barcelona, España: Editorial Sol 90.

Shoemaker, P. J. \& Reese, S. D. (1996). Mediating the message: Theories of influences on mass media content. Nueva York, EE.UU.: Longman Publishers.

Siegel, L. (2008). El mundo a través de una pantalla: ser humano en la era de la multitud digital. Barcelona, España: Tendencias Editores.

Steimberg, O. (2000). Naturaleza y cultura en el ocaso (triunfal) del periodismo amarillo. CIC. Cuadernos de Información y Comunicación, 5, 235-240. 
Valdettaro, S. (2009). Diarios: entre Internet, la desconfianza y los árboles muertos. En M. Carlón \& C. A. Scolari (Eds.), El fin de los medios masivos (pp. 47-67). Argentina: La Crujía.

Weaver, D. H. (1996). Journalists in comparative perspective: Backgrounds and professionalism. The Public, 3 (4), 83-91.

Weaver, D. H. (1998). The global journalist: News people around the world. Nueva Jersey, EE.UU.: Hampton Press.

Weaver, D. H. (1999). Las actitudes profesionales de los periodistas en un contexto global. Comunicación y Sociedad, XII (2), 33-45.

Weaver, D. H. \& Wilhoit, C. G. (1986). The American journalist: A portrait of U.S. news people and their work. Bloomington, IN, EE.UU.: Indiana University Press.

Weaver, D. H. \& Wilhoit, C. G. (1996). The American journalist in the 1990s: U.S. news people at the end of an era. Mahwah, NJ, EE.UU.: Lawrence Erlbaum Associates, Publishers.

Weaver, D. H., Beam, R.A., Brownlee, B.J., Voakes, P.S. \& Wilhoit, G. C. (2007). The American journalist in the 21st. Century. Mahwah, NJ, EE.UU.: Lawrence Erlbaum Associates Publishers.

Fecha de recepción: 30/05/2011. Aceptación: 27/07/2011 